Research in Astronomy and Astrophysics manuscript no.

(LTEX: 2020-0196.tex; printed on August 28, 2020; 0:45)

\title{
Searching for $\gamma$-ray emission from LOTAAS pulsars
}

\author{
Qi-Wei Lu ${ }^{1,2}$, Zhong-Xiang Wang ${ }^{3,1}$ and Yi Xing ${ }^{1}$ \\ ${ }^{1}$ Shanghai Astronomical Observatory, Chinese Academy of Sciences, Shanghai 200030, China; \\ wangzx@shao.ac.cn \\ ${ }^{2}$ Graduate University of the Chinese Academy of Sciences, Beijing 100049, China \\ ${ }^{3}$ Department of Astronomy, Yunnan University, Kunming 650091, China
}

\begin{abstract}
The LOw-Frequency ARray (LOFAR) has recently conducted a survey (LOFAR Tied-Array All-sky Survey; LOTAAS) for pulsars in the Northern hemisphere that resulted in discoveries of 73 new pulsars. For the purpose of studying the properties of these pulsars, we search for their $\gamma$-ray counterparts using the all-sky survey data obtained with the Large Area Telescope (LAT) onboard the Fermi Gamma-Ray Space Telescope (Fermi). We analyze the LAT data for 70 LOTAAS pulsars (excluding two millisecond pulsars and one with the longest known spin period of $23.5 \mathrm{~s}$ ). We find one candidate counterpart to PSR J1017+30, which should be searched for the $\gamma$-ray pulsation signal once its timing solution is available. For other LOTAAS pulsars, we derive their $0.3-500 \mathrm{GeV}$ flux upper limits. In order to compare the LOTAAS pulsars with the known $\gamma$-ray pulsars, we also derive the $0.3-500 \mathrm{GeV}$ $\gamma$-ray fluxes for 112 of the latter contained in the Fermi LAT fourth source catalog. Based on the properties of the $\gamma$-ray pulsars, we derive upper limits on the spin-down luminosities of the LOTAAS pulsars. The upper limits are not very constraining but help suggest that most of the LOTAAS pulsars probably have $<10^{33} \mathrm{erg} \mathrm{s}^{-1}$ spin-down luminosities and are not expected to be detectable with Fermi LAT.
\end{abstract}

Key words: stars: pulsars — stars: neutron — gamma rays: stars

\section{INTRODUCTION}

Since the discovery of the first radio pulsar signal in 1967 (Hewish et al., 1968), nearly 3000 pulsars have been found (Manchester et al., 2005), resulting from different surveys mainly at radio bands. Among them, approximately 2000 are "young" radio pulsars (in this paper, "young" pulsars are used to distinguish them from most of the others, the old "recycled" millisecond pulsars; MSPs). These pulsars are believed to be born in supernova explosions, while in total there are possibly $\sim 100,000$ of them in our Galaxy (e.g., Swiggum et al. 2014). The new generation radio telescopes such as the Five-hundred-meter Aperture 
to find and study most of these pulsars (e.g., Oian et al. 2019; Keane et al. 2015) and thus lead to a more complete understanding of the physical properties of the radio pulsar population.

Recently, a survey for radio pulsars in the Northern hemisphere at the very low frequency range of 119$151 \mathrm{MHz}$ was carried out with the LOw-Frequency ARray (LOFAR; van Haarlem et al. 2013). This LOFAR Tied-Array All-sky Survey (LOTAAS) has resulted in the discovery of 73 new radio pulsars (Sanidas et al., 2019). These LOTAAS pulsars probably represent a sample of pulsars that are bright at low frequencies and are found to have longer spin periods than the known young pulsar population. It is thus interesting to study their overall properties, checking if there are any other differences between them and the known young pulsar population or other pulsar groups based on survey methods.

From the beginning of the $\gamma$-ray astronomy, it has been learnt and pointed out that pulsars are potential high-energy objects with $\gamma$-rays emitted from the magnetosphere due to their high surface magnetic fields (e.g., Romani \& Yadigaroglu 1995). Observations conducted with The Fermi Gamma-Ray Space Telescope (Fermi), which was launched in 2008, have confirmed that pulsars are the dominant $\gamma$-ray sources in our Galaxy (Abdo et al., 2013). The Large Area Telescope (LAT) onboard Fermi has been scanning the whole sky at the energy band of $0.1-500 \mathrm{GeV}$ and thus far 253 pulsars, according to the latest results provided by the Fermi LAT Multiwavelength Coordinating Group (MCG) 1 , have been detected with pulsed $\gamma$-ray emission. Among them, 135 are young pulsars. The $\gamma$-ray properties of these pulsars allow a deep probe into the emission mechanism of pulsars (e.g., Pierbattista et al. 2012).

Taking advantage of the all-sky $\gamma$-ray data collected by LAT, we carried out the search for $\gamma$-ray counterparts to the LOTAAS pulsars. The results of any detection or upper limits provide constraints on properties of this very low-frequency pulsar sample. In this paper, we report the results from our search. Below in Section 2, we describe the analysis of the Fermi LAT data and provide the results. In Section 3, we discuss the implication of the results.

\section{LAT DATA ANALYSIS AND RESULTS}

\subsection{LAT data and source models}

LAT is a $\gamma$-ray imaging instrument that continuously scans the whole sky in the GeV band (Atwood et al., 2009). In the analysis, we selected $0.1-500 \mathrm{GeV}$ LAT events inside a $20^{\circ} \times 20^{\circ}$ region centered at the position of each of our targets. The time period of the LAT data was from 2008-08-04 15:43:36 (UTC) to 2019-08-14 02:23:15 (UTC). The updated Fermi Pass 8 database was used. Following the recommendations of the LAT tean 2 , we excluded the events with zenith angles larger than 90 degrees (to prevent the Earth's limb contamination) and with quality flags of 'bad'.

For each of the targets, we constructed a source model. The sources, which are listed in the Fermi LAT fourth source catalog (4FGL; Abdollahi et al. 2020) and within a 20 degree radius circular region from a target, were included in the source model. The spectral forms of the sources are provided in 4FGL. In our analysis, we set the spectral parameters of the sources within 5 degrees of a target as free parameters, and fixed the other parameters at their catalog values. The background Galactic and extragalactic diffuse spectral

1 https://confluence.slac.stanford.edu/display/GLAMCOG/Public+List+of+LAT-Detected+Gamma-Ray+Pulsars 
models gll_iem_v07.fits and the file iso_P8R3_SOURCE_V2_v1.txt, respectively, were also included in the source model, and the normalizations of the two models were set as free parameters.

\subsection{LOTAAS pulsars}

Among the 73 radio pulsars discovered by LOTAAS, two are MSPs and PSR J0250+58 has the longest spin period $(P=23.5 \mathrm{~s})$ among the known pulsars (Sanidas et al., 2019). We excluded these three pulsars from our target list; the former are presumably exceptions in the LOTAAS pulsar sample and the latter was studied in detail by Tan et al. (2018). Assuming power-law emission for each of the LOTAAS pulsars in the source models at their positions given in Sanidas et al. (2019), we performed standard binned likelihood analysis of the LAT data using Fermitools. To avoid the relatively large uncertainties of the instrument response function of LAT and the strong background emission (or possible contamination from nearby sources) in the Galactic plane in the low energy range of $<0.3 \mathrm{GeV}$, we included events in the energy range of $0.3-$ $500 \mathrm{GeV}$ for the likelihood analysis. We re-fit the source models using gtlike to the LAT data. With the fitted source models, the Test Statistic (TS) map of a $3^{\circ} \times 3^{\circ}$ region centered at each of the pulsar targets was calculated (using gttsmap). All the catalog sources were included in the source models. A TS value at a given position is a measurement of the fit improvement for including a source at the position, and is approximately the square of the detection significance of the source (Abdollahi et al., 2020). From the TS maps, we found only two possibly detected sources near the positions of PSR J1017+30 and PSR J1715+46. For the other 68 targets without any significantly detected sources at their positions, we derived 95\% flux upper limits, which are given in Table 1.

Table 1: 0.3-500 GeV flux upper limits of 68 LOTAAS pulsars.

\begin{tabular}{lccc}
\hline $\begin{array}{l}\text { Source } \\
\text { name }\end{array}$ & $\begin{array}{c}\text { R.A. } \\
(\mathrm{h}: \mathrm{m})\end{array}$ & $\begin{array}{c}\text { Decl. } \\
\left({ }^{\circ}:^{\prime}\right)\end{array}$ & $\begin{array}{c}\text { Flux } / 10^{-13} \\
\left(\mathrm{erg} \mathrm{cm}^{-2} \mathrm{~s}^{-1}\right)\end{array}$ \\
\hline J0039+35 & $00: 39.1$ & $+35: 45$ & 6.3 \\
$\mathrm{~J} 0059+69$ & $00: 59.5$ & $+69: 55$ & 14 \\
$\mathrm{~J} 0100+80$ & $01: 00.3$ & $+80: 22$ & 4.4 \\
$\mathrm{~J} 0107+13$ & $01: 07.6$ & $+13: 25$ & 3.1 \\
$\mathrm{~J} 0115+63$ & $01: 15.6$ & $+63: 24$ & 6.1 \\
$\mathrm{~J} 0121+14$ & $01: 22.0$ & $14: 16$ & 8.4 \\
$\mathrm{~J} 0139+33$ & $01: 40.0$ & $+33: 37$ & 2.0 \\
$\mathrm{~J} 0210+58$ & $02: 11.0$ & $+58: 44$ & 11 \\
$\mathrm{~J} 0302+22$ & $03: 02.5$ & $+22: 50$ & 1.5 \\
$\mathrm{~J} 0305+11$ & $03: 05.1$ & $+11: 23$ & 4.9 \\
J0317+13 & $03: 17.9$ & $+13: 29$ & 5.3 \\
$\mathrm{~J} 0349+23$ & $03: 49.9$ & $+23: 41$ & 15 \\
$\mathrm{~J} 0421+32$ & $04: 21.4$ & $+32: 54$ & 17 \\
$\mathrm{~J} 0454+45$ & $04: 54.9$ & $+45: 28$ & 15 \\
$\mathrm{~J} 0518+51$ & $05: 18.3$ & $+51: 25$ & 4.1
\end{tabular}


Q. Lu, Z. Wang, \& Y. Xing

\begin{tabular}{|c|c|c|c|}
\hline $\begin{array}{l}\text { Source } \\
\text { name }\end{array}$ & $\begin{array}{l}\text { R.A. } \\
\text { (h:m) }\end{array}$ & $\begin{array}{l}\text { Decl. } \\
\left({ }^{\circ}:^{\prime}\right) \\
\end{array}$ & $\begin{array}{c}\text { Flux } / 10^{-13} \\
\left(\operatorname{erg~cm}^{-2} \mathrm{~s}^{-1}\right)\end{array}$ \\
\hline $\mathrm{J} 0742+43$ & $07: 42.6$ & $+43: 33$ & 1.9 \\
\hline $\mathrm{J} 0811+37$ & $08: 11.2$ & $+37: 28$ & 1.8 \\
\hline $\mathrm{J} 0813+22$ & 08:13.9 & 22:01 & 2.2 \\
\hline $\mathrm{J} 0857+33$ & $08: 57.8$ & $+33: 48$ & 4.5 \\
\hline $\mathrm{J} 0928+30$ & 09:29.0 & $+30: 38$ & 3.9 \\
\hline $\mathrm{J} 0935+33$ & $09: 35.1$ & $+33: 11$ & 0.88 \\
\hline $\mathrm{J} 1226+00$ & $12: 26.2$ & $+00: 03$ & 7.1 \\
\hline $\mathrm{J} 1235-02$ & $12: 35.9$ & $-02: 05$ & 5.8 \\
\hline $\mathrm{J} 1303+38$ & $13: 03.3$ & $+38: 13$ & 4.1 \\
\hline $\mathrm{J} 1334+10$ & $13: 34.5$ & $+10: 05$ & 3.3 \\
\hline $\mathrm{J} 1344+66$ & $13: 43.9$ & $+66: 33$ & 14 \\
\hline $\mathrm{J} 1404+11$ & $14: 04.6$ & $+11: 57$ & 2.6 \\
\hline $\mathrm{J} 1426+52$ & $14: 27.0$ & $+52: 10$ & 1.7 \\
\hline $\mathrm{J} 1529+40$ & $15: 29.2$ & $+40: 49$ & 9.4 \\
\hline $\mathrm{J} 1623+58$ & $16: 23.8$ & $+58: 49$ & 1.3 \\
\hline $\mathrm{J} 1635+23$ & $16: 35.1$ & $+23: 31$ & 1.8 \\
\hline $\mathrm{J} 1638+40$ & $16: 38.8$ & $+40: 05$ & 8.5 \\
\hline $\mathrm{J} 1643+13$ & $16: 43.8$ & $+13: 25$ & 3.8 \\
\hline $\mathrm{J} 1655+62$ & $16: 55.9$ & $+62: 02$ & 2.0 \\
\hline $\mathrm{J} 1657+33$ & $16: 57.7$ & $+33: 03$ & 6.1 \\
\hline $\mathrm{J} 1707+35$ & $17: 07.0$ & $+35: 56$ & 6.5 \\
\hline $\mathrm{J} 1713+78$ & $17: 13.5$ & $+78: 09$ & 2.2 \\
\hline $\mathrm{J} 1722+35$ & $17: 22.1$ & $+35: 18$ & 3.9 \\
\hline $\mathrm{J} 1735+63$ & $17: 35.1$ & $+63: 19$ & 2.3 \\
\hline $\mathrm{J} 1740+27$ & $17: 40.5$ & $+27: 13$ & 8.5 \\
\hline $\mathrm{J} 1741+38$ & $17: 41.2$ & $+38: 54$ & 7.4 \\
\hline $\mathrm{J} 1745+12$ & $17: 45.7$ & $+12: 51$ & 3.3 \\
\hline $\mathrm{J} 1745+42$ & $17: 45.8$ & $+42: 53$ & 8.9 \\
\hline $\mathrm{J} 1749+59$ & $17: 49.6$ & $+59: 51$ & 1.5 \\
\hline $\mathrm{J} 1809+17$ & 18:09.1 & $+17: 04$ & 15 \\
\hline $\mathrm{J} 1810+07$ & $18: 10.7$ & $+07: 03$ & 2.9 \\
\hline $\mathrm{J} 1814+22$ & $18: 14.6$ & $+22: 23$ & 12 \\
\hline $\mathrm{J} 1848+15$ & $18: 48.9$ & $+15: 17$ & 4.9 \\
\hline $\mathrm{J} 1849+25$ & $18: 49.8$ & $+25: 58$ & 14 \\
\hline $\mathrm{J} 1910+56$ & $19: 10.7$ & $+56: 55$ & 0.82 \\
\hline $\mathrm{J} 1916+32$ & $19: 16.1$ & $+32: 24$ & 6.8 \\
\hline $\mathrm{J} 1933+53$ & $19: 33.0$ & $+53: 32$ & 2.7 \\
\hline
\end{tabular}




\begin{tabular}{lccc}
\hline $\begin{array}{l}\text { Source } \\
\text { name }\end{array}$ & $\begin{array}{c}\text { R.A. } \\
(\mathrm{h}: \mathrm{m})\end{array}$ & $\begin{array}{c}\text { Decl. } \\
\left({ }^{\circ}:^{\prime}\right)\end{array}$ & $\begin{array}{c}\text { Flux } / 10^{-13} \\
\left(\mathrm{erg} \mathrm{cm}^{-2} \mathrm{~s}^{-1}\right)\end{array}$ \\
\hline $\mathrm{J} 1953+30$ & $19: 53.8$ & $+30: 13$ & 3.0 \\
$\mathrm{~J} 1957-00$ & $19: 57.6$ & $-00: 01$ & 2.3 \\
$\mathrm{~J} 1958+56$ & $19: 58.0$ & $+56: 49$ & 8.3 \\
$\mathrm{~J} 2006+22$ & $20: 06.6$ & $+22: 04$ & 3.2 \\
$\mathrm{~J} 2022+21$ & $20: 22.4$ & $+21: 11$ & 2.3 \\
$\mathrm{~J} 2036+66$ & $20: 36.8$ & $+66: 44$ & 8.3 \\
$\mathrm{~J} 2051+12$ & $20: 51.4$ & $+12: 48$ & 2.8 \\
$\mathrm{~J} 2053+17$ & $20: 53.8$ & $+17: 18$ & 16 \\
$\mathrm{~J} 2057+21$ & $20: 57.8$ & $+21: 26$ & 14 \\
$\mathrm{~J} 2122+24$ & $21: 22.7$ & $+24: 24$ & 11 \\
$\mathrm{~J} 2123+36$ & $21: 23.8$ & $+36: 24$ & 3.4 \\
$\mathrm{~J} 2209+22$ & $22: 09.9$ & $+21: 17$ & 1.6 \\
$\mathrm{~J} 2306+31$ & $23: 06.2$ & $+31: 23$ & 6.1 \\
$\mathrm{~J} 2329+47$ & $23: 29.6$ & $+47: 42$ & 2.7 \\
$\mathrm{~J} 2336-01$ & $23: 36.6$ & $-01: 51$ & 12 \\
$\mathrm{~J} 2350+31$ & $23: 50.7$ & $+31: 39$ & 4.9 \\
\hline
\end{tabular}

\subsubsection{PSR J1017+30}

There are two $\gamma$-ray sources, clearly separated with each other, near the position of PSR J1017+30 with TS values of $\sim 22$ (Figure 1). We ran gtfindsrc in Fermitools to determine their positions and obtained R.A.=154.50, Decl.=30॰.18 (equinox J2000.0) for the northeast (NE) one and R.A. $=154.23$, Decl. $=29^{\circ} .85$ (equinox J2000.0) for the southwest (SW) one. The $1 \sigma$ nominal uncertainties are 0.05 and 0.04 , respectively. PSR J1017+30 is 0.09 away from the position of the NE source, but given the positional uncertainty of $3^{\prime}$ in the LOTAAS survey (Sanidas et al., 2019), the two sources match in position (see Figure 1), i.e., the NE source could be the $\gamma$-ray counterpart to PSR J1017+30.

Including the $\mathrm{NE}$ and $\mathrm{SW}$ sources in the source model, we re-performed the likelihood analysis to the $0.3-500 \mathrm{GeV}$ data, in which a power law was assumed for the two sources. Note that pulsars' $\gamma$ ray emission can generally be described with an exponentially cutoff power law (Abdo et al., 2013), but since the detection significance of the NE source was low, we chose to use a simple power law instead (we tested the former model, but no higher significant results were obtained). We obtained $\Gamma=2.4 \pm 0.3$ and $F_{0.3-500}=4 \pm 2 \times 10^{-10}$ photons s$~^{-1} \mathrm{~cm}^{-2}$ for the NE source with a TS value of $15(\Gamma=1.9 \pm 0.3$, $F_{0.3-500}=2 \pm 1 \times 10^{-10}$ photons s $\mathrm{s}^{-1} \mathrm{~cm}^{-2}$ for the SW source with a TS value of 16 ). We also tested to use the data from $0.1 \mathrm{GeV}, \Gamma$ was nearly the same but the flux was increased to $2 \pm 1 \times 10^{-9}$ photons s${ }^{-1} \mathrm{~cm}^{-2}$ and $\mathrm{TS} \simeq 19$ Therefore the NE source was detected at $\simeq 4 \sigma$.

We extracted the $\gamma$-ray spectra of the NE source by performing maximum likelihood analysis of the LAT data in 10 evenly divided energy bands in logarithm from $0.1-500 \mathrm{GeV}$. In the extraction, the spectral 
Table 2: Fermi LAT flux measurements for the sources at the positions of PSR J1017+30 and PSR J1715+46.

\begin{tabular}{|c|c|c|c|c|c|}
\hline \multirow[b]{2}{*}{$\begin{array}{l}E \\
(\mathrm{GeV})\end{array}$} & \multirow[b]{2}{*}{$\begin{array}{l}\text { Band } \\
(\mathrm{GeV})\end{array}$} & \multicolumn{2}{|c|}{ PSR J1017+30 } & \multicolumn{2}{|c|}{ PSR J1715+46 } \\
\hline & & $\begin{array}{c}F / 10^{-12} \\
\left(\mathrm{erg} \mathrm{cm}^{-2} \mathrm{~s}^{-1}\right)\end{array}$ & $\mathrm{TS}$ & $\begin{array}{c}F / 10^{-12} \\
\left(\mathrm{erg} \mathrm{cm}^{-2} \mathrm{~s}^{-1}\right)\end{array}$ & $\mathrm{TS}$ \\
\hline 0.15 & $0.1-0.2$ & $0.9 \pm 0.5$ & 9 & 1.6 & 2 \\
\hline 0.36 & $0.2-0.5$ & 0.8 & 2 & 0.7 & 4 \\
\hline 0.84 & $0.5-1.3$ & 0.3 & 2 & 0.2 & 0 \\
\hline 1.97 & $1.3-3.0$ & 0.2 & 2 & 0.2 & 2 \\
\hline 4.62 & $3.0-7.1$ & $0.14 \pm 0.08$ & 5 & 0.1 & 0 \\
\hline 10.83 & $7.1-16.6$ & 0.2 & 1 & 0.2 & 0 \\
\hline 25.37 & $16.6-38.8$ & 0.4 & 3 & $0.5 \pm 0.2$ & 17 \\
\hline 59.46 & $38.8-91.0$ & 0.3 & 0 & 0.3 & 0 \\
\hline 139.36 & $91.0-213.3$ & 0.8 & 0 & 2.0 & 2 \\
\hline 326.60 & $213.3-500.0$ & 1.9 & 0 & 1.6 & 0 \\
\hline
\end{tabular}

rameters of the sources were fixed at the values obtained from the above maximum likelihood analysis. For the results, we kept only spectral data points when TS is greater than 5 ( $>2 \sigma$ significance) and derived $95 \%$ flux upper limits otherwise. The flux and TS values of the spectral data points are provided in Table 2

Since pulsars' $\gamma$-ray emission is stable (Abdo et al., 2013), we checked the long-term variability of the NE source by calculating its variability index $\mathrm{TS}_{v a r}$. Following the procedure introduced in Nolan et al. (2012), we derived fluxes of 14 time bins for its $\gamma$-ray emission, with each bin containing 300-day data. If the fluxes are constant, $\mathrm{TS}_{v a r}$ would be distributed as $\chi^{2}$ with 13 degrees of freedom. Variable sources would be identified with $\mathrm{TS}_{v a r}$ larger than 27.7 (at a 99\% confidence level). The computed $\mathrm{TS}_{v a r}$ for the NE source is 8.1 , indicating that there was no significant long-term variability in its $\gamma$-ray emission.

\subsubsection{PSR J1715+46}

The $0.3-500 \mathrm{GeV} \gamma$-ray emission near the position of PSR J1715+46 only had TS 12 (left panel of Figure 2), but detailed analysis indicated that it was more significant in a high energy range of $>16 \mathrm{GeV}$. We thus calculated the $16-210 \mathrm{GeV}$ TS map of a $3^{\circ} \times 3^{\circ}$ region centered at PSR J1715+46 and a TS value of $\simeq 17$ was found (right panel of Figure 2). We ran gtfindsrc to determine the position and obtained R.A. $=258.91$, Decl. $=46^{\circ} .14$ (equinox J2000.0) with a $1 \sigma$ nominal uncertainty of 0.05 . PSR J1715+46 is 0.09 away from this position. Considering $3^{\prime}$ positional uncertainty of the radio position, the pulsar matches in position with the $\gamma$-ray source. Using the $\gamma$-ray position, we re-performed the likelihood analysis. The obtained results were $\Gamma=1.4 \pm 0.3, F_{0.3-500}=5 \pm 4 \times 10^{-11}$ photons s${ }^{-1} \mathrm{~cm}^{-2}$ in the $0.3-500 \mathrm{GeV}$ band (with a TS value of 14 ), or $\Gamma=2.2 \pm 0.2, F_{16-210}=1.1 \pm 0.5 \times 10^{-11}$ photons s $\mathrm{cm}^{-2}$ in the 16-210 GeV band (with a TS value of 16).

Similarly to analysis to the data of PSR J1017+30, we also extracted the $\gamma$-ray spectrum of PSR J1715+46 and searched for its long-term variability. The obtained spectral data points are provided in 


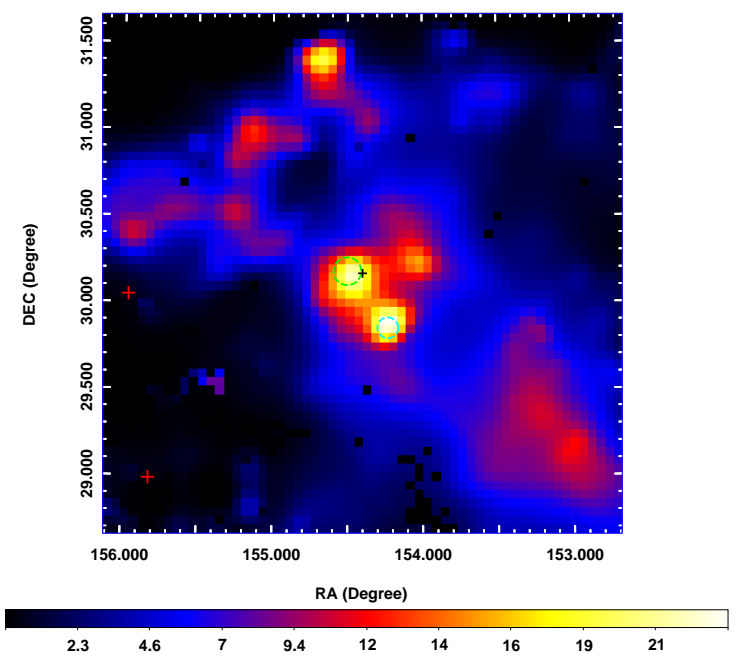

Fig. 1: $0.3-500 \mathrm{GeV}$ TS map of the $3^{\circ} \times 3^{\circ}$ region centered at PSR J1017+30. The image scale of the map is 0.05 pixel $^{-1}$. All 4FGL catalog sources (red pluses) were considered and removed. The black plus indicates the radio position with the length being the uncertainty of $3^{\prime}$. The two circles are the $2 \sigma$ error circles of the best-fit positions obtained for the two sources from Fermi analysis. The northeast source has a position consistent with that of PSR J1017+30.

value of 17. The computed $\mathrm{TS}_{\text {var }}$ for PSR J1715+46 in 16-210 GeV band is 15.3 , indicating that there was no significant long-term variability in the $\gamma$-ray source.
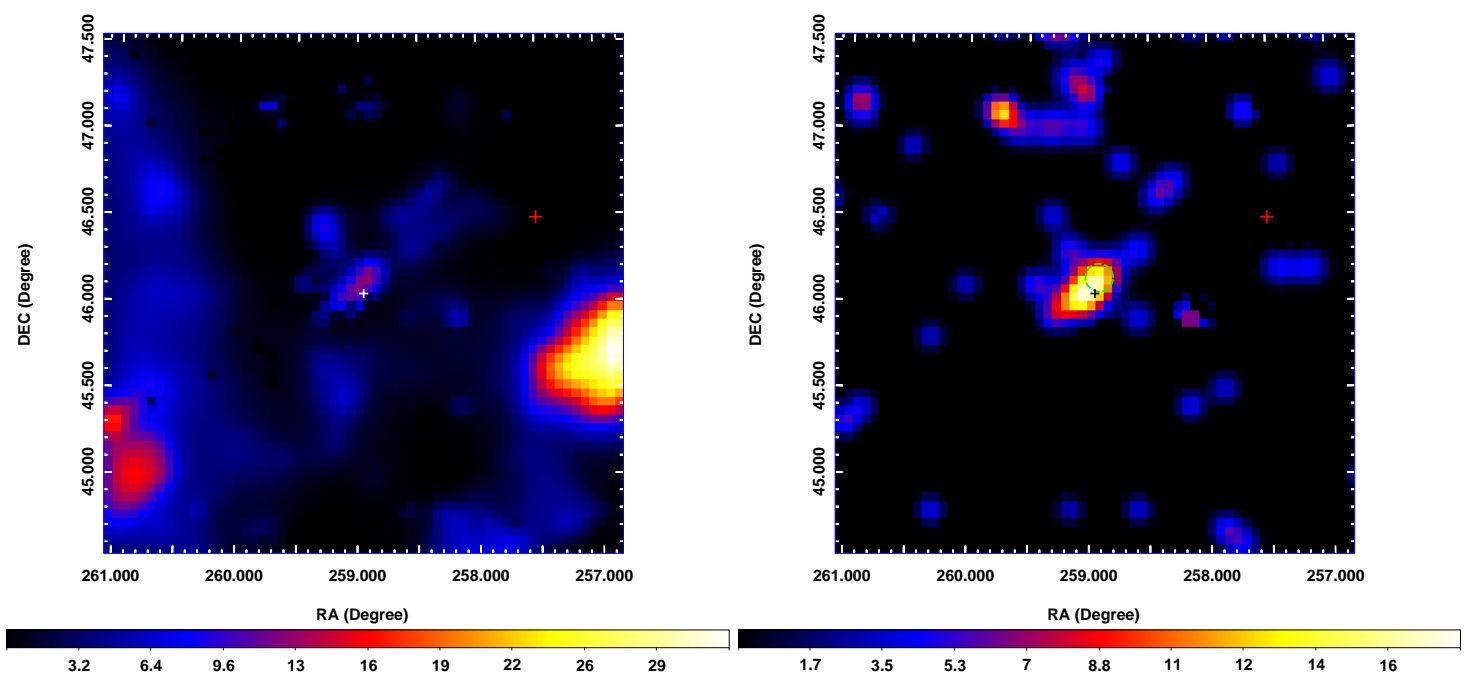

Fig. 2: TS maps of the $3^{\circ} \times 3^{\circ}$ region centered at PSR J1715+46 in the energy ranges of $0.3-500 \mathrm{GeV}$ (left) and $16-210 \mathrm{GeV}$ (right). The image scale of the maps is 0.05 pixel $^{-1}$. There is one catalog source, marked by a red plus, that was considered and removed. The white and black pluses mark the LOTAAS position of PSR J1715+46, with the length being the positional uncertainty of $3^{\prime}$. The green dashed circle in the right panel is the $2 \sigma$ error circle of the best-fit position obtained from Fermi analysis, indicating this $\gamma$-ray source 


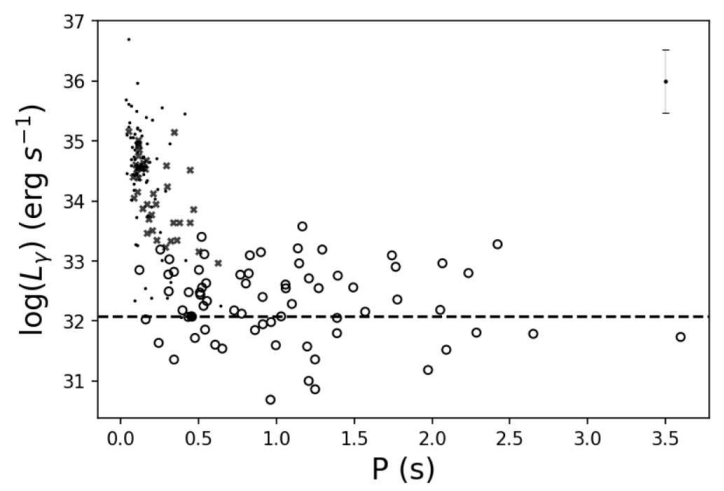

Fig. 3: $0.3-500 \mathrm{GeV}$ luminosities of the $\gamma$-ray pulsars (black dots and crosses, where the latter are $g u$ pulsars with questionable distances) and luminosity upper limits for the LOTAAS pulsars (open circles). PSR J1017+30 is marked as a filled circle. The indicative detection limit of the LAT survey is shown as the dashed line. A 30\% uncertainty is shown in the upper right conner to help indicate large uncertainties of the luminosities.

\section{DISCUSSION}

Having analyzed Fermi LAT data for 70 young pulsars discovered by LOTAAS, we have found that at the positions of two of them, PSR J1017+30 and PSR J1715+46, there was a $\gamma$-ray source respectively. The two sources were faint, and the detection significances were only $\simeq 4 \sigma$. For the $\gamma$-ray source to PSR J1715+46, our spectral analysis showed that the detection was mostly at the energy range of 16-39 GeV. Such emission is not consistent with the general $\gamma$-ray properties of pulsars, as most of their spectra have an exponential cutoff at several GeV (Abdo et al., 2013). Therefore we only suggest the $\gamma$-ray source at the position of PSR J1017+30 as a candidate counterpart. In the near future, once the timing solution of PSR J1017+30 is obtained, further data analysis to search for the pulsation signal at $\gamma$-rays would be able to verify if it is the counterpart. For PSR J1715+46, we derived its $0.3-500 \mathrm{GeV}$ flux upper limit, which is $2.6 \times$ $10^{-12} \operatorname{erg~cm}^{2} \mathrm{~s}^{-1}$.

Because of the lack of the period derivatives $\dot{P}$ of the LOTAAS pulsars, their properties such as the spin-down luminosities $\dot{E}$ and surface magnetic fields $B$ can not be estimated. In Figure 3 , we show $L_{\gamma}$ of the known $\gamma$-ray pulsars and the luminosity upper limits obtained for the LOTAAS pulsars, where for the former see appendix $\mathrm{A}$ and for the latter their distances were estimated from the dispersion measures (Sanidas et al., 2019). The former have $L_{\gamma} \geq 10^{32} \mathrm{erg} \mathrm{s}^{-1}$ and spin period $P \leq 0.6 \mathrm{~s}$, where 41 of the $g u$ pulsars have questionable distance values (marked with crosses in Figure 3, see appendix A). We note that the sensitivity limit of the Fermi LAT survey is approximately $10^{-12} \mathrm{erg} \mathrm{cm}^{-2} \mathrm{~s}^{-1}$ (Abdollahi et al., 2020), and assuming $1 \mathrm{kpc}$ distance for a pulsar, the luminosity limit would be $1.2 \times 10^{32} \mathrm{erg} \mathrm{s}^{-1}$. Thus the $\gamma$-ray pulsar detection has reached this indicative limit (Figure 3). For a few LOTAAS pulsars, the upper limits are below $10^{32} \mathrm{erg} \mathrm{s}^{-1}$, and several of them have $P \leq 0.6 \mathrm{~s}$. The comparison shows that our search is likely sensitive enough and should have been able to detect some of the LOTAAS pulsars if they have $\gamma$-ray emission similar to those of the $\gamma$-ray pulsars. It is interesting to note that PSR J1017+30 is right at 


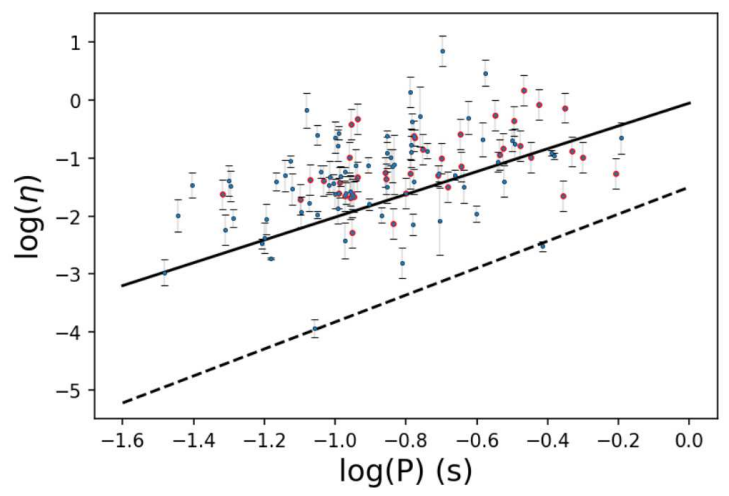

Fig. 4: $\log (\eta)$ and $\log (P)$ of the $\gamma$-ray pulsars (red data points are $g u$ pulsars with questionable distances). A relationship $\log (\eta)=1.97 \log (P)-0.05$ may describe the data points (solid line). The dashed line is $\log (\eta)=2.33 \log (P)-1.5$, which is a lower limit we define based on these pulsars.

For $\gamma$-ray pulsars, relationship $L_{\gamma} \sim \dot{E}^{1 / 2}$ is often considered (Pierbattista et al., 2012; Abdo et al., 2013). Thus $\gamma$-ray efficiency $\eta=L_{\gamma} / \dot{E} \sim \dot{E}^{-1 / 2}$. Since for pulsars $\dot{E} \sim \dot{P} / P^{3}$, and considering magnetic dipole radiation from pulsars (e.g., Lyne \& Graham-Smith 2012), $B \sim(P \dot{P})^{1 / 2}$, we can find $\dot{E} \sim B^{2} / P^{4}$ and $\eta \sim P^{2} / B$. In Figure 4 we show $\log (\eta)$ and $\log (P)$ of the $\gamma$-ray pulsars. A possible trend between them is seen. We test to fit the data points with function $\log (\eta)=a \log (P)+b$, and obtain $a \simeq 1.97$ and $b \simeq-0.05$, where $41 \mathrm{gu}$ pulsars with questionable distances were not included in the fitting. This possible trend is close to the relationship $\eta \sim P^{2}$ as well as $P^{2.33}$ predicted from considering curvature radiation from $\gamma$-ray pulsars (see details in Kalapotharakos et al. 2019), while note that $B$ is unknown and probably around $10^{12} \mathrm{G}$. However, since at any given $\log (P)$, there is a large scatter in $\log (\eta)$, the fit is not good (with reduced $\chi^{2} \sim 30$ ). We find that we may define a lower limit line to the data points with $\log (\eta)=2.33 \log (P)-1.5$ (Figure 4), for which we specifically require $\eta \sim P^{2.33}$ to match the theoretical prediction of curvature radiation from pulsars.

Using this lower limit, we may set constraints on $\dot{E}$ of the LOTAAS pulsars. Since $\eta=L_{\gamma} / \dot{E} \leq L_{\gamma}^{u} / \dot{E}$, where $L_{\gamma}^{u}$ is the $\gamma$-ray luminosity upper limits for the LOTAAS pulsars, we then have $\dot{E} \leq L_{\gamma}^{u} / \eta$. With the lower limit relationship given above, the upper limits on $\dot{E}$ of the LOTAAS pulsars are calculated, which are shown in Figure 5. In this calculation for PSR J1017+30, the luminosity of the candidate $\gamma$-ray counterpart is used. For comparison, we also include $\dot{E}$ of the $\gamma$-ray pulsars in the figure. The $\gamma$-ray pulsars generally have $\dot{E}>10^{33} \mathrm{erg} \mathrm{s}^{-1}$, and a death line around this value for $\gamma$-ray emission of pulsars has been considered (Wang \& Hirotani, 2011; Smith et al., 2019). Most of the LOTAAS pulsars have upper limits above this $\dot{E}$ value, and are mixed with the $\gamma$-ray pulsars in Figure 5 However because of the large scatters of $\log (\eta)$, for example, the solid line possibly describing $\log (\eta)$ and $\log (P)$ of the $\gamma$-ray pulsars in Figure 4 would increase $\eta 100$ times and thus lower $\dot{E}$ of the LOTAAS pulsars 100 times correspondingly, which will move most the LOTAAS pulsars below $10^{33} \mathrm{erg} \mathrm{s}^{-1}$. In addition, considering the indicative luminosity limit of the LAT survey for pulsars and still using this relationship, we have $\log (\dot{E})=31.79-2.35 \log (P)$, which is shown in Figure 5 Most of the LOTAAS pulsars will also be moved below this detection line. Therefore it is possible that most of the LOTAAS pulsars could have low $\dot{E}$ and thus low $\gamma$-ray emission, not to be 


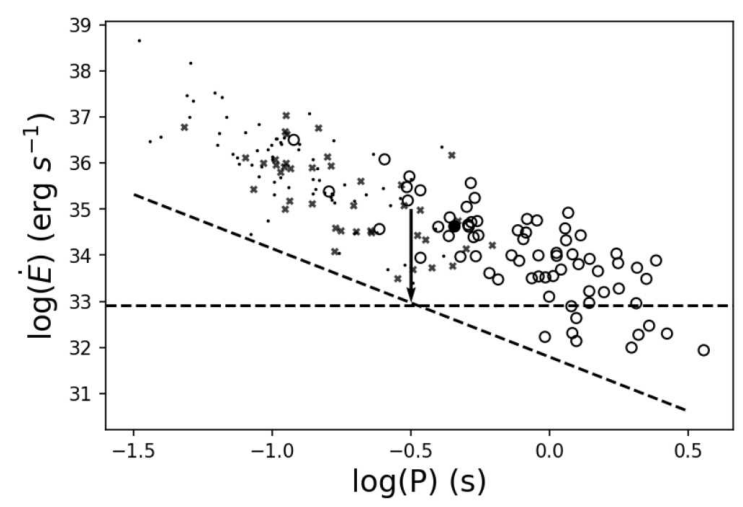

Fig. 5: Upper limits on $\dot{E}$ of LOTAAS pulsars (open circles), derived from lower limit line $\log (\eta) \geq$ $2.33 \log (P)-1.5$ (cf. Figure 4). These upper limits could be 100 times smaller (indicated by the arrow) because of the use of the lower limit line. An indicative detection limit of the LAT survey is shown as the dashed line (see the text for details). Values of $\dot{E}$ of the $\gamma$-ray pulsars (black dots and crosses) are also shown for comparison, and a dotted line of $8 \times 10^{32} \mathrm{erg} \mathrm{s}^{-1}$ is plotted to indicate a possible death line for $\gamma$-ray emission of pulsars (Smith et al., 2019). The filled circle marks PSR J1017+30.

As a summary, we have conducted analysis of the Fermi LAT data to search for $\gamma$-ray emission from the newly discovered 70 LOTAAS pulsars, and only found a candidate counterpart to one of them, PSR J1017+30. For this pulsar, once its long-term timing solution is obtained, a search for its pulsation signal in the $\gamma$-ray emission of the candidate can be conducted for the purpose of verifying if it is the $\gamma$ ray counterpart. We provide the $0.3-500 \mathrm{GeV}$ flux upper limits for the non-detections. By comparing the LOTAAS pulsars with the known $\gamma$-ray pulsars, we estimate the $\dot{E}$ upper limits for the LOTAAS pulsars. The upper limits are not very constraining, and it is likely that most of the LOTAAS pulsars have low, $<10^{33} \mathrm{erg} \mathrm{s}^{-1} \dot{E}$ and are not expected to have detectable $\gamma$-ray emission.

Acknowledgements We thank anonymous referees for very helpful suggestions. This research made use of the High Performance Computing Resource in the Core Facility for Advanced Research Computing at Shanghai Astronomical Observatory. This research was supported by the National Program on Key Research and Development Project (Grant No. 2016YFA0400804) and the National Natural Science Foundation of China (11633007, U1738131). Z.W. acknowledges the support by the Original Innovation Program of the Chinese Academy of Sciences (E085021002).

\section{Appendix A: KNOWN $\gamma$-RAY PULSARS}

There are 121 young $\gamma$-ray pulsars listed in 4FGL (Abdollahi et al., 2020). Searching in the Australia Telescope National Facility (ATNF) pulsar catalog (Manchester et al., 2005) and references provided at MCG, we found distance values for 112 of them, which are given in Table A.1 However it can be noted that 53 of $121 \gamma$-ray pulsars are marked with ' $g u$ ' at MCG, which indicates that these pulsars were discovered in LAT data and/or using an LAT seed position. As most of the $g u$ pulsars have not been detected at radio frequencies, they are probably assigned with 'heuristic' distances (see Abdo et al. 2013). We searched references for $g u$ pulsars in Table A.1 and found no radio detection reported for 41 of them. To be cautious, 
Using the spectral parameters given in 4FGL, we derived $0.3-500 \mathrm{GeV}$ flux $F_{0.3-500}$. For the pulsars without distance uncertainties, we assumed 30\% uncertainties (e.g., Camilo et al. 2009). We calculated the luminosities from $L_{\gamma}=4 \pi d^{2} f_{\Omega} F_{0.3-500}$, where $d$ is the distance and $f_{\Omega}$ is the beam correction factor. Following Abdo et al. (2013), we adopted $f_{\Omega}=1$.

Table A.1: $0.3-500 \mathrm{GeV}$ fluxes and luminosities for $112 \gamma$-ray pulsars.

\begin{tabular}{|c|c|c|c|c|c|}
\hline $\begin{array}{l}\text { Source } \\
\text { name }\end{array}$ & $\begin{array}{l}\mathrm{P} \\
(\mathrm{s})\end{array}$ & $\begin{array}{c}\text { Distance }^{a} \\
\quad(\mathrm{kpc})\end{array}$ & $\begin{array}{c}\dot{E} / 10^{33} \\
\left(\mathrm{erg} \mathrm{s}^{-1}\right)\end{array}$ & $\begin{array}{c}\text { Flux } / 10^{-12} \\
\left(\mathrm{erg} \mathrm{cm}^{-2} \mathrm{~s}^{-1}\right)\end{array}$ & $\begin{array}{l}\text { Luminosity } \\
\left(10^{33} \mathrm{erg} \mathrm{s}^{-1}\right)\end{array}$ \\
\hline $\mathrm{J} 0002+6216^{g}$ & 0.12 & 6.36 & 150 & $14.7 \pm 1.2$ & $71 \pm 43$ \\
\hline $\mathrm{J} 0007+7303$ & 0.32 & $1.4 \pm 0.3$ & 450 & $387.2 \pm 3.1$ & $91 \pm 39$ \\
\hline J0106+4855 & 0.083 & $3.1 \pm 1.1$ & 29 & $17.5 \pm 0.8$ & $20 \pm 14$ \\
\hline J0205+6449 & 0.066 & $1.95 \pm 0.04$ & 27000 & $40.8 \pm 1.1$ & $49.9 \pm 2.5$ \\
\hline J0248+6021 & 0.22 & $2.0 \pm 0.2$ & 210 & $22.5 \pm 1.3$ & $10.8 \pm 2.2$ \\
\hline $\mathrm{J} 0357+3205^{g}$ & 0.44 & 0.83 & 5.9 & $53.1 \pm 1.0$ & $4.4 \pm 2.6$ \\
\hline $\mathrm{J} 0359+5414^{g}$ & 0.079 & 3.45 & 1300 & $17.7 \pm 1.3$ & $25 \pm 15$ \\
\hline J0514-4408 & 0.32 & 0.97 & 2.5 & $3.9 \pm 0.3$ & $0.43 \pm 0.27$ \\
\hline $\mathrm{J} 0534+2200$ & 0.033 & $2 \pm 0.5$ & 460000 & $1000 \pm 17$ & $490 \pm 240$ \\
\hline J0540-6919 & 0.051 & 49.7 & 150000 & $17.0 \pm 1.1$ & $5030 \pm 3034$ \\
\hline $\mathrm{J} 0554+3107^{g}$ & 0.46 & 1.9 & 56 & $17.0 \pm 0.7$ & $7.3 \pm 4.4$ \\
\hline $\mathrm{J} 0622+3749^{g}$ & 0.33 & 1.6 & 27 & $14.3 \pm 0.6$ & $4.4 \pm 2.6$ \\
\hline $\mathrm{J} 0631+0646^{g}$ & 0.11 & 4.58 & 100 & $15.3 \pm 1.4$ & $38 \pm 23$ \\
\hline J0631+1036 & 0.29 & $1.0 \pm 0.2$ & 170 & $27.9 \pm 1.2$ & $14.7 \pm 5.9$ \\
\hline $\mathrm{J} 0633+0632^{g}$ & 0.30 & 1.35 & 120 & $80.9 \pm 2.3$ & $18 \pm 11$ \\
\hline J0633+1746 & 0.24 & $0.25 \pm 0.08$ & 32 & $3700 \pm 11$ & $16 \pm 10$ \\
\hline $\mathrm{J} 0659+1414$ & 0.38 & $0.29 \pm 0.03$ & 38 & $11.3 \pm 0.4$ & $0.114 \pm 0.024$ \\
\hline J0729-1448 & 0.25 & $3.5 \pm 0.4$ & 280 & $3.6 \pm 0.8$ & $3.1 \pm 1.0$ \\
\hline J0742-2822 & 0.17 & $2.1 \pm 0.5$ & 140 & $11.6 \pm 1.0$ & $5.5 \pm 2.7$ \\
\hline J0835-4510 & 0.089 & $0.28 \pm 0.02$ & 6900 & $7700 \pm 27$ & $72 \pm 10$ \\
\hline J0908-4913 & 0.11 & $2.6 \pm 0.9$ & 490 & $15.5 \pm 2.5$ & $2.5 \pm 1.3$ \\
\hline J0940-5428 & 0.088 & $3.0 \pm 0.5$ & 1900 & $12.6 \pm .9$ & $0.218 \pm 0.074$ \\
\hline J1016-5857 & 0.11 & $2.9 \pm 0.6$ & 2600 & $54.2 \pm 3.6$ & $65 \pm 27$ \\
\hline J1019-5749 & 0.16 & 10.91 & 180 & $17.3 \pm 2.3$ & $250 \pm 150$ \\
\hline $\mathrm{J} 1023-5746^{g}$ & 0.11 & 2.08 & 11000 & $111.7 \pm 6.9$ & $58 \pm 35$ \\
\hline J1028-5819 & 0.091 & $2.3 \pm 0.3$ & 840 & $204.2 \pm 4.7$ & $49 \pm 13$ \\
\hline $\mathrm{J} 1044-5737^{g}$ & 0.14 & 1.9 & 800 & $82.3 \pm 2.6$ & $36 \pm 21$ \\
\hline J1048-5832 & 0.12 & $2.7 \pm 0.4$ & 2000 & $150.4 \pm 3.3$ & $150 \pm 91$ \\
\hline $\mathrm{J} 1055-6028$ & 0.10 & 3.83 & 1200 & $16.6 \pm 1.7$ & $29 \pm 18$ \\
\hline J1057-5226 & 0.20 & $0.3 \pm 0.2$ & 30 & $252.8 \pm 2.3$ & $0.24 \pm 0.33$ \\
\hline $\mathrm{J} 1057-5851^{g}$ & 0.62 & 0.8 & 17 & $12.01 \pm 0.88$ & $0.92 \pm 0.56$ \\
\hline
\end{tabular}




\begin{tabular}{|c|c|c|c|c|c|}
\hline $\begin{array}{l}\text { Source } \\
\text { name }\end{array}$ & $\begin{array}{l}\mathrm{P} \\
(\mathrm{s})\end{array}$ & $\begin{array}{c}\text { Distance }^{a} \\
(\mathrm{kpc})\end{array}$ & $\begin{array}{c}\dot{E} / 10^{33} \\
\left(\mathrm{erg} \mathrm{s}^{-1}\right)\end{array}$ & $\begin{array}{c}\text { Flux } / 10^{-12} \\
\left(\mathrm{erg} \mathrm{cm}^{-2} \mathrm{~s}^{-1}\right)\end{array}$ & $\begin{array}{c}\text { Luminosity } \\
\left(10^{33} \mathrm{erg} \mathrm{s}^{-1}\right)\end{array}$ \\
\hline J1105-6107 & 0.063 & $5 \pm 1$ & 2500 & $15.7 \pm 2.0$ & $10.4 \pm 4.4$ \\
\hline J1112-6103 & 0.064 & 4.5 & 4500 & $16.4 \pm 2.8$ & $40 \pm 25$ \\
\hline J1119-6127 & 0.41 & $8.4 \pm 0.4$ & 2300 & $33.5 \pm 2.1$ & $280 \pm 32$ \\
\hline J1124-5916 & 0.14 & $4.8 \pm 0.7$ & 12000 & $41.6 \pm 1.6$ & $120 \pm 37$ \\
\hline J1151-6108 & 0.10 & 2.22 & 390 & $9.04 \pm 0.93$ & $5.3 \pm 3.2$ \\
\hline $\mathrm{J} 1208-6238^{g}$ & 0.44 & 3 & 1500 & $30.8 \pm 2.5$ & $33 \pm 20$ \\
\hline $\mathrm{J} 1253-5820$ & 0.26 & 1.64 & 5 & $3.26 \pm 0.99$ & $1.05 \pm 0.71$ \\
\hline J1341-6220 & 0.19 & 12.6 & 1400 & $16.9 \pm 2.8$ & $320 \pm 199$ \\
\hline $\mathrm{J} 1350-6225^{g}$ & 0.14 & 1.3 & 130 & $36.9 \pm 2.3$ & $7.4 \pm 4.5$ \\
\hline J1357-6429 & 0.17 & $2.5 \pm 0.5$ & 3100 & $19.3 \pm 1.3$ & $22.22 \pm 0.90$ \\
\hline J1410-6132 & 0.050 & $15.6 \pm 4.2$ & 10000 & $18.9 \pm 4.7$ & $410 \pm 240$ \\
\hline $\mathrm{J} 1413-6205^{g}$ & 0.11 & 2.15 & 830 & $155.3 \pm 4.8$ & $86 \pm 52$ \\
\hline $\mathrm{J} 1418-6058^{g}$ & 0.11 & $1.6 \pm 0.7$ & 4900 & $240 \pm 12$ & $100 \pm 91$ \\
\hline $\mathrm{J} 1420-6048$ & 0.068 & $5.6 \pm 0.9$ & 10000 & $100 \pm 11$ & $390 \pm 130$ \\
\hline $\mathrm{J} 1422-6138^{g}$ & 0.34 & 4.8 & 96 & $51.9 \pm 2.7$ & $140 \pm 86$ \\
\hline $\mathrm{J} 1429-5911^{g}$ & 0.12 & 1.95 & 770 & $81.0 \pm 4.3$ & $37 \pm 22$ \\
\hline $\mathrm{J} 1459-6053^{g}$ & 0.10 & 1.84 & 910 & $86.2 \pm 2.3$ & $35 \pm 21$ \\
\hline $\mathrm{J} 1509-5850$ & 0.089 & $2.6 \pm 0.5$ & 520 & $97.2 \pm 2.8$ & $130 \pm 51$ \\
\hline $\mathrm{J} 1522-5735^{g}$ & 0.10 & 2.1 & 1200 & $55.8 \pm 2.6$ & $29 \pm 18$ \\
\hline $\mathrm{J} 1528-5838^{g}$ & 0.36 & 1.1 & 22 & $15.4 \pm 1.3$ & $2.2 \pm 1.4$ \\
\hline $\mathrm{J} 1531-5610$ & 0.084 & $2.1 \pm 0.3$ & 900 & $15.7 \pm 1.9$ & $15.1 \pm 9.3$ \\
\hline J1614-5048 & 0.23 & 5.15 & 1600 & $16.2 \pm 4.4$ & $52 \pm 34$ \\
\hline $\mathrm{J} 1623-5005^{g}$ & 0.085 & 1.3 & 270 & $5.6 \pm 3.3$ & $11.4 \pm 6.9$ \\
\hline $\mathrm{J} 1624-4041^{g}$ & 0.17 & 1.8 & 39 & $23.1 \pm 1.1$ & $8.9 \pm 5.4$ \\
\hline J1648-4611 & 0.16 & $4.5 \pm 0.7$ & 210 & $37.7 \pm 2.2$ & $90 \pm 28$ \\
\hline $\mathrm{J} 1702-4128$ & 0.18 & $4.8 \pm 0.6$ & 340 & $24.2 \pm 4.0$ & $45 \pm 14$ \\
\hline J1705-1906 & 0.30 & 0.75 & 6.1 & $3.64 \pm 0.46$ & $0.24 \pm 0.15$ \\
\hline J1709-4429 & 0.10 & $2.3 \pm 0.3$ & $1153.2 \pm 8.0$ & $930 \pm 240$ & \\
\hline J1718-3825 & 0.075 & $3.6 \pm 0.4$ & 1300 & $79.7 \pm 4.8$ & $120 \pm 27$ \\
\hline $\mathrm{J} 1730-3350$ & 0.14 & 3.49 & 1200 & $26.1 \pm 4.9$ & $38 \pm 24$ \\
\hline $\mathrm{J} 1732-3131$ & 0.20 & $0.6 \pm 0.1$ & 150 & $166.7 \pm 3.4$ & $8.2 \pm 2.7$ \\
\hline J1739-3023 & 0.11 & 3.07 & 300 & $20.0 \pm 3.2$ & $23 \pm 14$ \\
\hline $\mathrm{J} 1740+1000$ & 0.15 & 1.23 & 230 & $1.95 \pm 0.37$ & $0.35 \pm 0.22$ \\
\hline J1741-2054 & 0.41 & $0.38 \pm 0.02$ & 9.5 & $96.3 \pm 1.9$ & $1.04 \pm 0.11$ \\
\hline $\mathrm{J} 1746-3239^{g}$ & 0.20 & 0.8 & 33 & $42.5 \pm 2.6$ & $3.3 \pm 2.0$ \\
\hline $\mathrm{J} 1747-2958$ & 0.10 & $4.8 \pm 0.8$ & 2500 & $121.3 \pm 6.7$ & $92.1 \pm 31.1$ \\
\hline $\mathrm{J} 1801-2451$ & 0.12 & $5.2 \pm 0.5$ & 2600 & $24.2 \pm 2.6$ & $41.8 \pm 9.2$ \\
\hline
\end{tabular}




\begin{tabular}{|c|c|c|c|c|c|}
\hline $\begin{array}{l}\text { Source } \\
\text { name }\end{array}$ & $\begin{array}{l}\mathrm{P} \\
(\mathrm{s})\end{array}$ & $\begin{array}{c}\text { Distance }^{a} \\
(\mathrm{kpc})\end{array}$ & $\begin{array}{c}\dot{E} / 10^{33} \\
\left(\mathrm{erg} \mathrm{s}^{-1}\right)\end{array}$ & $\begin{array}{c}\text { Flux } / 10^{-12} \\
\left(\mathrm{erg} \mathrm{cm}^{-2} \mathrm{~s}^{-1}\right)\end{array}$ & $\begin{array}{l}\text { Luminosity } \\
\left(10^{33} \mathrm{erg} \mathrm{s}^{-1}\right)\end{array}$ \\
\hline $\mathrm{J} 1803-2149^{g}$ & 0.11 & 1.3 & 640 & $69.8 \pm 3.8$ & $14.1 \pm 8.5$ \\
\hline $\mathrm{J} 1809-2332$ & 0.15 & $1.7 \pm 1.0$ & 430 & $362.3 \pm 6.2$ & $34 \pm 39$ \\
\hline $\mathrm{J} 1813-1246^{g}$ & 0.048 & 2.63 & 6200 & $177.2 \pm 4.1$ & $150 \pm 88$ \\
\hline $\mathrm{J} 1826-1256$ & 0.11 & 1.55 & 3600 & $340.0 \pm 8.8$ & $98 \pm 59$ \\
\hline $\mathrm{J} 1827-1446^{g}$ & 0.50 & 0.7 & 14 & $24.7 \pm 1.7$ & $1.44 \pm 0.88$ \\
\hline $\mathrm{J} 1828-1101$ & 0.072 & 4.77 & 1600 & $29.6 \pm 5.0$ & $80.3 \pm 50.1$ \\
\hline J1833-1034 & 0.062 & $4.7 \pm 0.4$ & 34000 & $56.4 \pm 4.6$ & $110 \pm 21$ \\
\hline $\mathrm{J} 1836+5925$ & 0.17 & $0.5 \pm 0.3$ & 11 & $537.7 \pm 2.9$ & $5.8 \pm 7.0$ \\
\hline $\mathrm{J} 1837-0604$ & 0.096 & 4.77 & 2000 & $35.2 \pm 5.8$ & $96 \pm 60$ \\
\hline $\mathrm{J} 1838-0537^{g}$ & 0.15 & 2 & 5900 & $9.1 \pm 1.0$ & $44 \pm 27$ \\
\hline $\mathrm{J} 1844-0346^{g}$ & 0.11 & 4.3 & 4300 & $41.8 \pm 2.8$ & $92 \pm 56$ \\
\hline $\mathrm{J} 1846+0919^{g}$ & 0.23 & 1.53 & 34 & $32.0 \pm 1.0$ & $8.9 \pm 5.4$ \\
\hline J1853-0004 & 0.10 & 5.34 & 210 & $10.2 \pm 1.7$ & $35 \pm 22$ \\
\hline $\mathrm{J} 1857+0143$ & 0.14 & 4.57 & 450 & $22.3 \pm 3.6$ & $56 \pm 35$ \\
\hline $\mathrm{J} 1906+0722^{g}$ & 0.11 & 1.91 & 1000 & $55.5 \pm 5.6$ & $24 \pm 15$ \\
\hline $\mathrm{J} 1907+0602$ & 0.11 & $3.2 \pm 0.3$ & 2800 & $240.9 \pm 5.2$ & $160 \pm 31$ \\
\hline J1913+0904 & 0.16 & 3 & 160 & $19.3 \pm 1.9$ & $21 \pm 13$ \\
\hline $\mathrm{J} 1913+1011$ & 0.036 & 4.61 & 2900 & $11.5 \pm 2.5$ & $29 \pm 19$ \\
\hline $\mathrm{J} 1925+1720$ & 0.076 & 5.06 & 950 & $9.1 \pm 1.5$ & $28 \pm 17$ \\
\hline $\mathrm{J} 1932+1916^{g}$ & 0.21 & 1.5 & 410 & $48.9 \pm 3.5$ & $13.1 \pm 7.9$ \\
\hline $\mathrm{J} 1932+2220$ & 0.14 & 10.9 & 750 & $3.84 \pm 0.98$ & $55 \pm 36$ \\
\hline $\mathrm{J} 1935+2025$ & 0.08 & 4.6 & 4600 & $21.5 \pm 1.9$ & $54 \pm 33$ \\
\hline $\mathrm{J} 1952+3252$ & 0.039 & $2.0 \pm 0.5$ & 3700 & $118.4 \pm 2.3$ & $120 \pm 64$ \\
\hline $\mathrm{J} 1954+2836^{g}$ & 0.092 & 1.96 & 1000 & $8.8 \pm 2.9$ & $41 \pm 24$ \\
\hline $\mathrm{J} 1957+5033^{g}$ & 0.37 & 1.36 & 5.3 & $20.1 \pm 0.63$ & $4.4 \pm 2.7$ \\
\hline $\mathrm{J} 1958+2846^{g}$ & 0.29 & 1.95 & 340 & $86.3 \pm 2.8$ & $39 \pm 24$ \\
\hline $\mathrm{J} 2006+3102$ & 0.16 & 6.03 & 220 & $8.7 \pm 1.4$ & $38 \pm 24$ \\
\hline $\mathrm{J} 2017+3625^{g}$ & 0.17 & 0.656 & 12 & $56.8 \pm 3.8$ & $2.9 \pm 1.8$ \\
\hline $\mathrm{J} 2021+3651$ & 0.10 & $10 \pm 2$ & 3400 & $424.1 \pm 5.4$ & $160 \pm 66$ \\
\hline $\mathrm{J} 2022+3842$ & 0.08 & 4.6 & 4700 & $14.5 \pm 2.1$ & $170 \pm 110$ \\
\hline $\mathrm{J} 2021+4026$ & 0.27 & $1.5 \pm 0.4$ & 120 & $645.5 \pm 8.8$ & $360 \pm 190$ \\
\hline $\mathbf{J} 2028+3332^{g}$ & 0.18 & 0.9 & 35 & $51.5 \pm 1.5$ & $5.0 \pm 3.0$ \\
\hline $\mathrm{J} 2030+3641$ & 0.20 & $3 \pm 1$ & 32 & $39.2 \pm 1.8$ & $230 \pm 140$ \\
\hline $\mathrm{J} 2030+4415^{g}$ & 0.23 & 0.72 & 32 & $36.7 \pm 1.5$ & $2.3 \pm 1.4$ \\
\hline $\mathrm{J} 2032+4127$ & 0.14 & $3.7 \pm 0.6$ & 270 & $130.7 \pm 3.8$ & $27.6 \pm 9.0$ \\
\hline $\mathrm{J} 2043+2740$ & 0.096 & $1.8 \pm 0.3$ & 56 & $7.22 \pm 0.52$ & $1.89 \pm 0.64$ \\
\hline $\mathrm{J} 2055+2539^{g}$ & 0.32 & 0.62 & 4.9 & $46.59 \pm 0.96$ & $2.1 \pm 1.3$ \\
\hline
\end{tabular}




\begin{tabular}{lccccc}
\hline $\begin{array}{l}\text { Source } \\
\text { name }\end{array}$ & $\begin{array}{c}\mathrm{P} \\
(\mathrm{s})\end{array}$ & $\begin{array}{c}\text { Distance }^{a} \\
(\mathrm{kpc})\end{array}$ & $\begin{array}{c}\dot{E} / 10^{33} \\
\left(\mathrm{erg} \mathrm{s}^{-1}\right)\end{array}$ & $\begin{array}{c}\text { Flux/10 } \\
\left(\mathrm{erg} \mathrm{cm}^{-2} \mathrm{~s}^{-1}\right)\end{array}$ & $\begin{array}{c}\text { Luminosity } \\
\left(10^{33} \mathrm{erg} \mathrm{s}^{-1}\right)\end{array}$ \\
\hline $\mathrm{J} 2111+4606^{g}$ & 0.16 & 2.7 & 1400 & $39.6 \pm 1.2$ & $35 \pm 21$ \\
$\mathrm{~J} 2139+4716^{g}$ & 0.28 & 0.8 & 3.1 & $22.48 \pm 0.98$ & $1.7 \pm 1.0$ \\
$\mathrm{~J} 2208+4056$ & 0.64 & 0.75 & 0.81 & $2.68 \pm 0.43$ & $0.18 \pm 0.11$ \\
$\mathrm{~J} 2229+6114$ & 0.052 & $0.8 \pm 0.15$ & 22000 & $187.8 \pm 2.7$ & $200 \pm 76$ \\
$\mathrm{~J} 2238+5903^{g}$ & 0.16 & 2.83 & 890 & $49.7 \pm 1.6$ & $47.6 \pm 28.6$ \\
$\mathrm{~J} 2240+5832$ & 0.14 & $7.7 \pm 0.7$ & 220 & $8.4 \pm 1.0$ & $53 \pm 12$ \\
\hline
\end{tabular}

${ }^{a}$ Distances without errors are assumed to have $30 \%$ uncertainties.

${ }^{g} \gamma$-ray pulsars without (reported) radio detection.

\section{References}

Abdo, A. A., Ajello, M., Allafort, A., et al. 2013, ApJS, 208, 17

Abdollahi, S., Acero, F., Ackermann, M., et al. 2020, ApJS, 247, 33

Atwood, W. B., Abdo, A. A., Ackermann, M., et al. 2009, ApJ, 697, 1071

Camilo, F., Ng, C. Y., Gaensler, B. M., et al. 2009, ApJ, 703, L55

Hewish, A., Bell, S. J., Pilkington, J. D. H., Scott, P. F., \& Collins, R. A. 1968, Nature, 217, 709

Kalapotharakos, C., Harding, A. K., Kazanas, D., et al. 2019, ApJ, 883, L4

Keane, E., Bhattacharyya, B., Kramer, M., et al. 2015, in Advancing Astrophysics with the Square

Kilometre Array (AASKA14), 40

Lyne, A., \& Graham-Smith, F. 2012, Pulsar Astronomy

Manchester, R. N., Hobbs, G. B., Teoh, A., \& Hobbs, M. 2005, AJ, 129, 1993

Nolan, P. L., Abdo, A. A., Ackermann, M., et al. 2012, ApJS, 199, 31

Pierbattista, M., Grenier, I. A., Harding, A. K., \& Gonthier, P. L. 2012, A\&A, 545, A42

Qian, L., Pan, Z., Li, D., et al. 2019, Science China Physics, Mechanics, and Astronomy, 62, 959508

Romani, R. W., \& Yadigaroglu, I. A. 1995, ApJ, 438, 314

Sanidas, S., Cooper, S., Bassa, C. G., et al. 2019, A\&A, 626, A104

Smith, D. A., Bruel, P., Cognard, I., et al. 2019, ApJ, 871, 78

Swiggum, J. K., Lorimer, D. R., McLaughlin, M. A., et al. 2014, ApJ, 787, 137

Tan, C. M., Bassa, C. G., Cooper, S., et al. 2018, ApJ, 866, 54

van Haarlem, M. P., Wise, M. W., Gunst, A. W., et al. 2013, A\&A, 556, A2

Wang, R., \& Hirotani, K. 2011, ApJ, 736, 127 